\title{
Mechanical evaluation for laminated bamboo lumber along two eccentric compression directions
}

\author{
Hai-tao $\mathrm{Li}^{1,2} \cdot$ Gang $\mathrm{Wu}^{2} \cdot$ Qi-sheng $\mathrm{Zhang}^{1} \cdot$ Jing-Wen $\mathrm{Su}^{3}$
}

Received: 18 June 2016/Accepted: 25 August 2016/Published online: 20 September 2016

(C) The Japan Wood Research Society 2016

\begin{abstract}
To evaluate the mechanical performance under two eccentric compression directions, 80 laminated bamboo lumber (LBL) column specimens were tested and analysed. Mechanical properties along two directions were compared. Bamboo nodes and drill hole are two main failure reasons for the specimens under two eccentric directions. More crack layers appeared for radial eccentric direction group specimens due to the layer structure. No matter which eccentric directions they are, the lateral deflection curves are close to the sine line, and the strain across the cross section of the LBL column for each specimen is basically linear throughout the loading process, following standard normal section bending theory. The mechanical properties for two directions are similar with each other and could follow the same design rules. Although the mechanical properties are similar for both LBL columns and parallel bamboo strand lumber (PBSL) columns as a whole, there are still some clear performance differences between the two kinds of materials. Combined all the test data under two eccentric directions, an equation for calculating the radial eccentricity influencing coefficient $\varphi_{\mathrm{e}}$ of LBL columns is proposed. The calculation
\end{abstract}

Hai-tao Li

lhaitao1982@126.com

1 Collage of Civil Engineering, Nanjing Forestry University (Southeast University, Key Laboratory of Concrete and PreStressed Concrete Structure of Ministry of Education), Nanjing 210037, China

2 College of Civil Engineering, Southeast University, Nanjing 210096, China

3 Nanjing Technical Vocational College, Nanjing 210019, China results obtained from the equations give a good agreement with the test results.

Keywords Laminated bamboo lumber - Column - Radial eccentric compression - Tangential eccentric direction . Eccentricity ratio

\section{Introduction}

As a promising wood substitute, bamboo's structural forms in which it can be used are limited due to the diameter of the bamboo culm and the low rigidity of the bamboo. To solve these problems, modern industrial bamboo materials appeared in different forms, such as Glubam [1, 2], parallel bamboo strand lumber (PBSL) [3, 4], laminated bamboo lumber (LBL) [5-10], and so on. Bamboo tubes can be disassembled into bamboo filament bundles by passing them through a roller press crusher and then gluing it together with adhesive to form certifiable structural members. The composite material is called PBSL [3, 4]. As for LBL [5-10], original bamboo tubes can be disassembled into thin flat laminae first and then laminated together with adhesive to form certifiable structural members. Both the cross section and length can be controlled freely in the factory, and thus, LBL is competitive with commonly used building materials, whilst also having renewable characteristics.

Basic mechanical performances, such as tensile, shear, compressive, and bending, have been investigated by many researchers [5-23] through small specimens, considering effects of layer structure, bamboo species, oil treatment, loading direction, processing methods, and glue type, etc, while there are not too many studies on structural members. Li et al. [13], Sharma et al. [18], Lee et al. [20], Wei 
et al. [21], Lima Douglas Mateus de et al. [22], and Sinha et al. [23] have examined the bending properties of LBL beams separately in a structural size, and a good research conclusion has been given.

However, less research reports could be found for the LBL columns. Li et al. [24] have studied the buckling performance of PBSL columns under axial compression. Taheri Li et al. [25] investigated the buckling response of glue-laminated columns reinforced with fibre-reinforced plastic sheets. The study of $\mathrm{Li}$ et al. [26] showed that the short LBL columns (with the cross section of $100 \mathrm{~mm} \times 100 \mathrm{~mm}$ ) in compression display a significant amount of plastic behaviour before crushing, and also showed that the stress-strain relationship in compression could be represented using a tri-linear model with an elastic portion, and elastoplastic portion and a purely plastic portion. Su et al. [27] investigated the mechanical performance of laminated bamboo lumber column pier under axial compression. Luna et al. [28] and Li et al. [29] have investigated the mechanical performance of laminated bamboo column under axial compression considering the slenderness ratio, respectively. Li et al. [3] have investigated the eccentric compression performance of PBSL column based on 45 specimens, and the designed eccentricity values are $0,10,25,40,55,70,80,90,100$, and $120 \mathrm{~mm}$. Li et al. [30] have investigated the mechanical properties of $35 \mathrm{LBL}$ column specimens with a design dimension of $73 \mathrm{~mm} \times 73 \mathrm{~mm} \times 1000 \mathrm{~mm}$ under radial eccentric compression, and the test specimens have mechanical connections. As discussed above, except for $\mathrm{Li}$ et al. [30] 's study, it is difficult to find papers addressing the performance of LBL columns under eccentric compression.

To the best of our knowledge, a few studies on LBL in compression have been conducted under eccentric compression conditions. However, almost all columns used in the building industry experience eccentric compression. A few studies have been performed under eccentric compression for bamboo lumber pieces. Due to the low shear strength of the material and the complicated test setup, it is not easy to perform the eccentric compression test. The behaviour of structural members under eccentric compression could be significantly different from its behaviour under axial compression. This study aims to examine the tangential and radial eccentric compression behaviour of LBL structural members and investigate how the eccentricity ratio influences the behaviour of LBL columns under two eccentric compression directions on the basis of larger test specimen number.

As we all know, bamboo is one kind of anisotropic materials, and different directions have different mechanical properties. What is the mechanical performance along different directions after laminating those strips together?
In addition, are there any mechanical property differences between the LBL and PBSL columns under eccentric condition? These are interesting questions. To know the eccentric compression behaviour of LBL columns under tangential and radial deviation directions, the study will examine in detail the behaviour of full size structural members (with a design dimension of $100 \mathrm{~mm} \times 100 \mathrm{~mm} \times 1200 \mathrm{~mm}$ ) constructed from laminated bamboo lumber with different eccentricity ratios. Detailed comparisons between the mechanical properties along two directions for the LBL columns will be investigated in the following, as well as the comparisons between the LBL and PBSL columns [3] under eccentric condition.

\section{Materials and test methods}

Harvested at the age of 3-4 years, the source Moso bamboo (Phyllostachys pubescens) was chosen from Fujian province. The cross section of bamboo strips elements for LBL is rectangle (Fig. 1) with the dimension of $8 \mathrm{~mm} \times 21 \mathrm{~mm}$. The length for all strips is the same as the column specimens, which means that there are no mechanical connections along the longitudinal direction for all strips. Following the same measures as mentioned by $\mathrm{Li}$ et al. [26] before, laminated bamboo lumbers were made finally. The final moisture content was $6.52 \%$, and the density was $640.25 \mathrm{~kg} / \mathrm{m}^{3}$ for the materials. According to the compression tests, the compression strength for the laminated bamboo is $80.43 \mathrm{MPa}$, with the modulus of elasticity of 9694.3 MPa, Poisson's ratio of 0.33 .

Bamboo has three main directions, which are longitudinal, radial, and tangential. Considering two eccentric directions (Fig. 1), respectively, and different eccentricity values of $0,10,25,40,55,70,80,90,100,110$, and

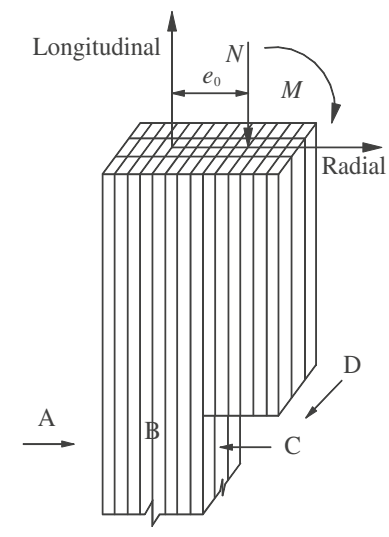

(a) Radial direction

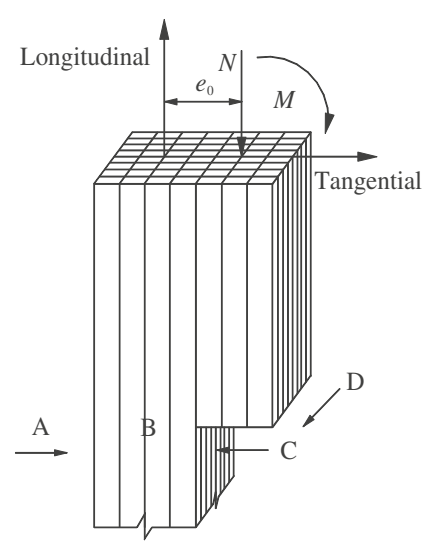

(b) Tangential direction
Fig. 1 Specimen and eccentric direction sketch 
$120 \mathrm{~mm}$, twenty groups of specimens, with the same cross section of $100 \mathrm{~mm} \times 100 \mathrm{~mm}$ and the same length of $1200 \mathrm{~mm}$, were constructed. Each group consisted of four identical specimens, and the total number is 80 for all the specimens. In addition, the specimen groups are named by 'LBCC + eccentricity'. One example was given in the following to differentiate the specimens which directions they are. The number suffixed following ' $\mathrm{LBCC}+\mathrm{ec}$ centricity' tells which eccentric direction they are. For example, the specimen from LBCC10-1 to LBCC10-4 is under radial eccentric direction, while the specimen from LBCC10-5 to LBCC10-8 is under tangential eccentric direction.

The displacements for axial direction, the quarter points, including the mid-span deflection were measured by three laser displacement sensors (LDS model: Keyence IL-300), respectively. Two strain gauges were pasted on each middle side surface of the specimens except one side surface (face D) with six train gauges, and the strain gauges were numbered, as shown in Fig. 2. The load is along the eccentricity line which is parallel to the axial line. The test was performed using a microcomputer-controlled electrohydraulic servo universal testing machine with a capacity of $1000 \mathrm{kN}$ and a data acquisition system (model: TDS530).

The total loading time is controlled between 8 and $12 \mathrm{~min}$. The load was applied initially through load control in the elastic stage, and then was changed to displacement control before the proportional limit. The test continued at a certain displacement rate until the load reduced by $15 \%$ of the ultimate load or the middle deflection value is about $40 \mathrm{~mm}$ or so after the peak load point or the specimen had sustained significant damage, at which time testing was halted.

\section{Test results and analysis}

\section{Failure modes and mechanism analysis}

The main test results can be seen from Table 1 except group LBCC0. Stable failure happened for this group under axial compression, and the ultimate load values for group LBCC0 are 509.9, 537.0, 510.7, and $5144 \mathrm{kN}$. The mean ultimate load for this group is $518.0 \mathrm{kN}$ with a standard deviation of $12.85 \mathrm{kN}$ and a coefficient of variation of $2.5 \%$. Based on the mean and standard deviation quoted, the characteristic ultimate load for these specimens is 496.8 $\mathrm{kN}$.

All specimens for both eccentric directions behaved elastically at the beginning of loading. With the increase of loading, the specimens showed a small amount plastic deformation and the stiffness of the column decreased significantly. Then, cracks (accompanied by a slight noise) appeared on the tensile surface A as the deflection became obvious. Finally, the load reduced by $15 \%$ of the ultimate load or the specimen had sustained significant damage or the middle deflection value is too big. The columns shed most of the load at this time, and can be considered to have failed. Except for face C, cracks can be seen clearly from other three side surface. Bending failure always happens for all the column specimens under two eccentric directions compression.

On the basis of the first crack appearing position and damage process, three typical failure modes can be classified, respectively, for two eccentric directions. Typical failure modes for radial eccentric direction can be seen from Fig. 3. The first one is named failure mode I (Fig. 3a) which the specimen split first around the

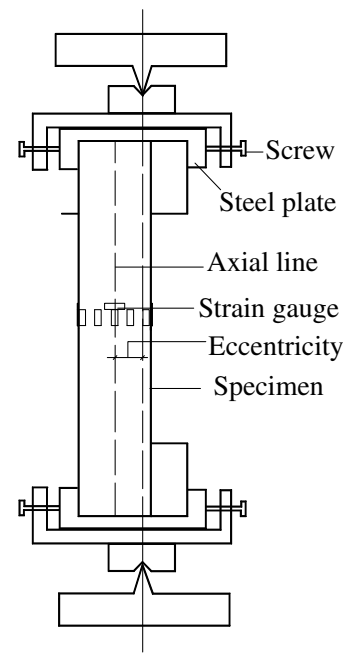

(a) Test scheme

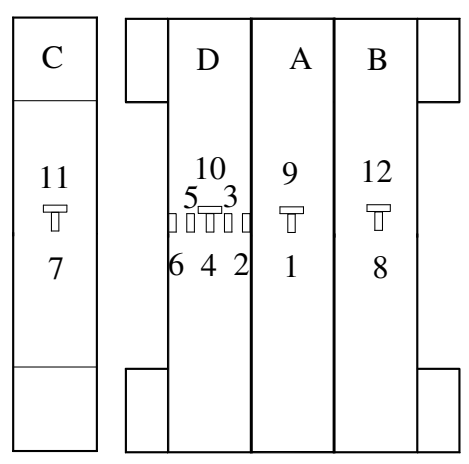

(b) Strain gauges arrangement

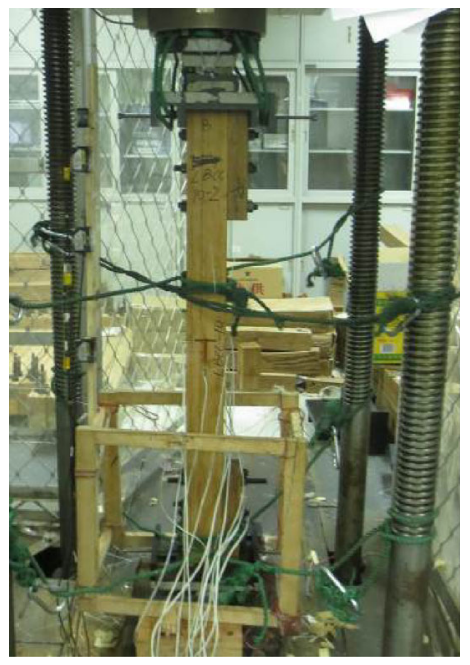

(c) Test photos

Fig. 2 Eccentric compressive test for columns 
Table 1 Test results

\begin{tabular}{|c|c|c|c|c|c|c|c|c|}
\hline Specimen & $N_{\mathrm{ul}}(\mathrm{kN})$ & $w_{\mathrm{ul}}(\mathrm{mm})$ & $\varepsilon_{\text {uasA }}(\mu \varepsilon)$ & $\varepsilon_{\text {uasC }}(\mu \varepsilon)$ & $\varepsilon_{\mathrm{ulsA}}(\mu \varepsilon)$ & $\varepsilon_{\mathrm{ulsC}}(\mu \varepsilon)$ & $s_{\mathrm{ul}}(\mathrm{mm})$ & $M_{\mathrm{ul}}(\mathrm{kN} \mathrm{m})$ \\
\hline LBCC10-1 & 230.0 & 20.9 & 3227 & -9910 & -973 & 2944 & 9.49 & 7.12 \\
\hline LBCC10-2 & 228.4 & 20.1 & 2273 & -8900 & -817 & 3248 & 12.2 & 6.87 \\
\hline LBCC10-3 & 230.1 & 21.4 & 3094 & -7045 & -876 & 3036 & 9.76 & 7.22 \\
\hline LBCC10-4 & 228.7 & 18.3 & 2297 & -8644 & -770 & 2581 & 12.0 & 6.46 \\
\hline LBCC10-5 & 227.7 & 21.5 & 3534 & -9700 & -940 & 3045 & 8.04 & 7.16 \\
\hline LBCC10-6 & 232.0 & 35.9 & 2445 & -8055 & -696 & 2625 & 8.49 & 10.6 \\
\hline LBCC10-7 & 234.3 & 18.6 & 2914 & -9008 & -766 & 2280 & 7.83 & 6.70 \\
\hline LBCC10-8 & 223.2 & 23.0 & 3788 & -10568 & -962 & 2945 & 8.34 & 7.36 \\
\hline LBCC25-1 & 178.1 & 37.2 & 7443 & -13067 & -1921 & 4229 & 11.3 & 11.1 \\
\hline LBCC $25-2$ & 182.8 & 40.2 & 8379 & -16075 & -2141 & 4158 & 12.8 & 11.9 \\
\hline LBCC $25-3$ & 186.0 & 44.5 & 9319 & -17030 & -2742 & 5188 & 13.9 & 12.9 \\
\hline LBCC25-4 & 182.3 & 44.5 & 7709 & -10129 & -2397 & 6248 & 12.8 & 12.7 \\
\hline LBCC $25-5$ & 182.6 & 40.5 & 7237 & -14516 & -2022 & - & 14.3 & 12.0 \\
\hline LBCC25-6 & 180.5 & 35.3 & 6493 & -13004 & -1714 & 3318 & 12.2 & 10.9 \\
\hline LBCC25-7 & 188.7 & 42.8 & 8281 & -13946 & -2054 & 5432 & 13.2 & 12.8 \\
\hline LBCC25-8 & 180.5 & 40.2 & 7848 & -15069 & -2141 & 4481 & 13.1 & 11.8 \\
\hline LBCC40-1 & 148.8 & 46.6 & 10975 & -15845 & -2772 & 2831 & 23.8 & 12.9 \\
\hline LBCC40-2 & 149.4 & 52.6 & 10698 & -15301 & -2929 & 6597 & 24.7 & 13.8 \\
\hline LBCC40-3 & 138.3 & 46.6 & 10046 & -16591 & -2808 & 4859 & 21.6 & 12.0 \\
\hline LBCC40-4 & 140.7 & 48.5 & 8904 & -17060 & -2885 & 5082 & 19.1 & 12.5 \\
\hline LBCC40-5 & 145.4 & 43.5 & 10677 & -18656 & -2674 & 5602 & 23.3 & 12.1 \\
\hline LBCC40-6 & 151.9 & 53.0 & 11001 & -18602 & -2498 & 5398 & 22.6 & 14.1 \\
\hline LBCC40-7 & 137.1 & 39.9 & 9206 & -14225 & -2530 & 4036 & 16.0 & 10.9 \\
\hline LBCC40-8 & 137.2 & 33.1 & 6808 & -10882 & -1934 & 3048 & 14.4 & 10.0 \\
\hline LBCC55-1 & 127.5 & 47.7 & 10227 & -14540 & -3141 & 4810 & 22.9 & 13.1 \\
\hline LBCC55-2 & 131.4 & 50.0 & 10847 & -18153 & -2642 & 6082 & 23.7 & 13.8 \\
\hline LBCC55-3 & 125.7 & 47.2 & 9385 & -15544 & -2375 & 4734 & 22.6 & 12.8 \\
\hline LBCC55-4 & 129.4 & 54.8 & 11500 & -15082 & -3407 & 5738 & 29.2 & 14.2 \\
\hline LBCC55-5 & 124.9 & 41.2 & 7416 & -12925 & -2085 & 3825 & 17.6 & 12.0 \\
\hline LBCC55-6 & 117.5 & - & 8662 & -12818 & -2253 & 4324 & 19.2 & 6.46 \\
\hline LBCC55-7 & 130.2 & 45.8 & 10351 & -16510 & -3234 & 5107 & 24.6 & 13.1 \\
\hline LBCC55-8 & 127.9 & 39.9 & 8062 & -13155 & -2278 & 4375 & 19.2 & 12.1 \\
\hline LBCC70-1 & 105.9 & 37.0 & 7860 & -10198 & -2207 & 3422 & 19.8 & 11.3 \\
\hline LBCC70-2 & 107.4 & 42.0 & 9035 & -13906 & -2674 & 4290 & 20.6 & 12.0 \\
\hline LBCC70-3 & 112.4 & 45.0 & 9011 & -14721 & -2995 & 4948 & 24.1 & 12.9 \\
\hline LBCC70-4 & 112.3 & 48.0 & 10319 & - & -2641 & 5836 & 25.9 & 13.3 \\
\hline LBCC70-5 & 107.3 & 38.5 & 8199 & -10911 & -2220 & 3798 & 21.0 & 11.6 \\
\hline LBCC70-6 & 108.3 & 47.8 & 9769 & -13932 & -2365 & 3746 & 26.7 & 12.8 \\
\hline LBCC70-7 & 100.2 & 38.1 & 8251 & -10094 & - & 3551 & 20.7 & 10.8 \\
\hline LBCC70-8 & 112.0 & 47.8 & 9900 & -15311 & -2581 & 5250 & 26.0 & 13.2 \\
\hline LBCC80-1 & 105.7 & 44.4 & 9444 & -11460 & -2964 & 4899 & 26.0 & 13.1 \\
\hline LBCC80-2 & 104.4 & 49.2 & 10708 & -14157 & -3070 & 5380 & 30.6 & 13.5 \\
\hline LBCC80-3 & 107.7 & 54.4 & 10322 & -16309 & -3225 & 5662 & 29.8 & 14.5 \\
\hline LBCC $80-4$ & 101.2 & 42.9 & 8910 & -14469 & -2564 & 4649 & 23.0 & 12.4 \\
\hline LBCC80-5 & 102.7 & 49.3 & 9881 & -14152 & -3000 & - & 30.5 & 13.3 \\
\hline LBCC80-6 & 99.3 & 40.1 & 8102 & -13452 & -1947 & 4019 & 22.3 & 11.9 \\
\hline LBCC80-7 & 95.1 & 36.3 & - & -9011 & -1386 & 3530 & 20.1 & 11.1 \\
\hline LBCC80-8 & 101.8 & 45.6 & 9485 & -11995 & -2683 & 4234 & 26.8 & 12.8 \\
\hline
\end{tabular}


Table 1 continued

\begin{tabular}{|c|c|c|c|c|c|c|c|c|}
\hline Specimen & $N_{\mathrm{ul}}(\mathrm{kN})$ & $w_{\mathrm{ul}}(\mathrm{mm})$ & $\varepsilon_{\text {uasA }}(\mu \varepsilon)$ & $\varepsilon_{\text {uasC }}(\mu \varepsilon)$ & $\varepsilon_{\mathrm{ulsA}}(\mu \varepsilon)$ & $\varepsilon_{\mathrm{ulsC}}(\mu \varepsilon)$ & $s_{\mathrm{ul}}(\mathrm{mm})$ & $M_{\mathrm{ul}}(\mathrm{kN} \mathrm{m})$ \\
\hline LBCC90-1 & 81.8 & 31.0 & 6010 & -6987 & -1601 & 2269 & 16.3 & 9.90 \\
\hline LBCC90-2 & 91.5 & 36.0 & 8447 & -9309 & -2032 & 2163 & 26.7 & 11.5 \\
\hline LBCC $90-3$ & 85.3 & 41.0 & 8219 & -5826 & -2196 & 3872 & 24.0 & 11.2 \\
\hline LBCC90-4 & 86.9 & 40.0 & 8966 & -10151 & -2248 & 3931 & 26.6 & 11.3 \\
\hline LBCC90-5 & 88.0 & 38.1 & 8124 & -11478 & -2222 & 3885 & 28.6 & 11.3 \\
\hline LBCC90-6 & 93.7 & 45.9 & 8014 & -10170 & -2152 & 3130 & 25.4 & 12.7 \\
\hline LBCC90-7 & 89.5 & 48.7 & 9852 & -14340 & -2731 & 3647 & 33.2 & 12.4 \\
\hline LBCC90-8 & 86.8 & 39.5 & 8197 & -11708 & - & 3499 & 25.8 & 11.2 \\
\hline LBCC100-1 & 76.8 & 41.0 & 7666 & -8586 & -1929 & 3519 & 31.4 & 10.8 \\
\hline LBCC100-2 & 81.6 & 39.1 & 8292 & -8132 & -2450 & 3381 & 29.3 & 11.4 \\
\hline LBCC100-3 & 84.6 & 44.5 & 8942 & -10243 & -2845 & 3569 & 30.9 & 12.2 \\
\hline LBCC100-4 & 79.3 & 40.6 & 8442 & -9840 & - & 4685 & 28.3 & 11.1 \\
\hline LBCC100-5 & 79.5 & 34.5 & 7268 & -8742 & -2018 & 2627 & 22.5 & 10.7 \\
\hline LBCC100-6 & 93.4 & 52.3 & 10366 & -12265 & -3259 & 5150 & 36.1 & 14.2 \\
\hline LBCC100-7 & 79.7 & - & 7647 & -10224 & -2133 & 3516 & 26.5 & 7.97 \\
\hline LBCC100-8 & 80.2 & 31.5 & 8227 & -8486 & -2392 & 3006 & 27.5 & 10.5 \\
\hline LBCC110-1 & 73.5 & 41.0 & 8123 & -12112 & -2520 & 3409 & 35.4 & 11.1 \\
\hline LBCC110-2 & 83.7 & 39.0 & 8193 & -13437 & - & 4660 & 34.5 & 12.5 \\
\hline LBCC110-3 & 84.2 & 39.0 & 10683 & -15449 & -2923 & 5460 & 35.9 & 12.5 \\
\hline LBCC110-4 & 80.8 & 40.0 & 9114 & -10071 & -2310 & 5457 & 34.1 & 12.1 \\
\hline LBCC110-5 & 80.7 & 42.2 & 8670 & -10965 & -3225 & 3198 & 34.1 & 12.3 \\
\hline LBCC110-6 & 80.6 & 41.0 & 8157 & -9545 & -2237 & 3570 & 27.5 & 12.2 \\
\hline LBCC110-7 & 80.0 & 39.6 & 8514 & -10621 & -2418 & 2652 & 28.9 & 12.0 \\
\hline LBCC110-8 & 80.4 & 37.8 & 8446 & -11263 & -2202 & 3095 & 26.9 & 11.9 \\
\hline LBCC120-1 & 72.1 & 45.8 & 8202 & -12178 & -2623 & 3418 & 36.4 & 12.0 \\
\hline LBCC120-2 & 80.2 & 48.6 & 9195 & -13881 & -2612 & 4941 & 42.1 & 13.5 \\
\hline LBCC120-3 & 73.8 & 51.9 & 7353 & -14095 & -2099 & 3489 & 41.9 & 12.7 \\
\hline LBCC120-4 & 78.5 & 49.4 & 8866 & -10596 & -2548 & 4289 & 39.8 & 13.3 \\
\hline
\end{tabular}

$N_{\mathrm{ul}}$ is the ultimate load. $w_{\mathrm{ul}}$ is the deflection for the peak load point. $\varepsilon_{\mathrm{uas} A}$ and $\varepsilon_{\mathrm{uasC}}$ are the longitudinal strain for face C and face A, respectively. $\varepsilon_{\mathrm{ulsA}}$ and $\varepsilon_{\mathrm{ulsC}}$ are the lateral strain for face $\mathrm{C}$ and face $\mathrm{A}$, respectively. $s_{\mathrm{ul}}$ is the axial displacement. $M_{\mathrm{ul}}$ is the ultimate moment

central position in face $\mathrm{A}$ (tensile side) and then the cracks appeared layer by layer mainly from tensile side to inner part of the specimen with the increase of loading. Most of the test specimens failed following the first mode. The cracks in both the central position and the position close to the bracket for failure mode II (Fig. 3b) appeared almost at the same time in the tensile surface (face A) and then the specimen split layer by layer close to the compression side. The third one is called failure mode III which the specimen split around the drill hole first in the tensile surface (face A), but the cracks did not extend on and along face $\mathrm{A}$. It can be seen clearly that there is none crack on the middle part of surface A, but several layers' cracks can be seen from face B. Figure 3c shows the third final failure mode.

Figure 4 shows the typical failure modes for tangential eccentric direction. Similar as radial direction, the specimen for failure mode I (Fig. 4a) split first around the central position in face A (tensile side), and then the cracks extended to the inner part of the specimen with the increase of loading. Most of the test specimens failed following the first mode. The cracks around the drill holes in the bracket part for failure mode II (Fig. 4b) appeared first in the tensile surface (face A) and extended along the cross section. The bracket part of the specimen was broken and almost fell off. None cracks can be seen in the central part of the specimen during the whole loading process. Only two specimens damaged like this which are LBCC100-5 and LBCC110-5. Similar as failure mode II, the specimen for failure mode III split around the drill hole firstly in the tensile surface (face A) and the cracks did not extend on and along face $\mathrm{A}$, but one main crack developed to the inner and middle part of the specimen. There is none crack on the middle part of surface A, but one main crack can be seen from face D clearly. Figure $4 c$ shows the third final failure mode. 


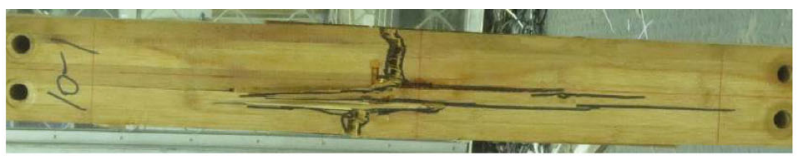

Face A

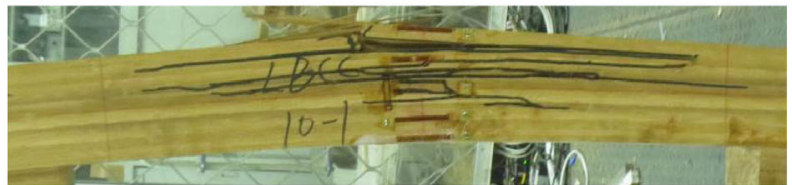

Face D

(a) Failure mode I (LBCC10-1)

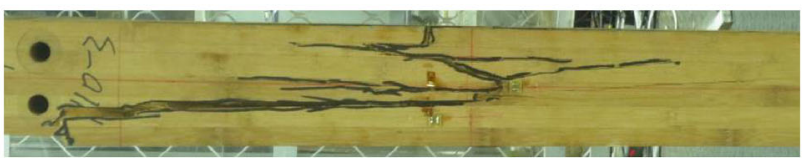

Face A

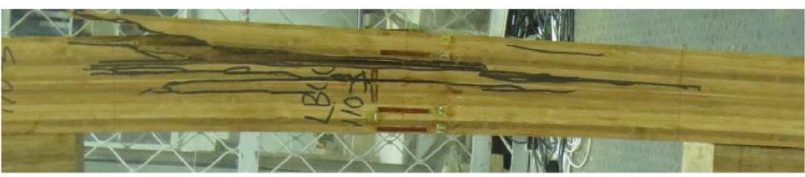

Face D

(b) Failure mode II (LBCC110-3)

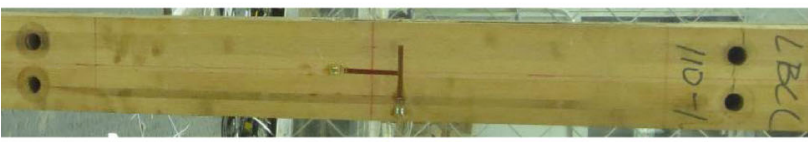

Face A

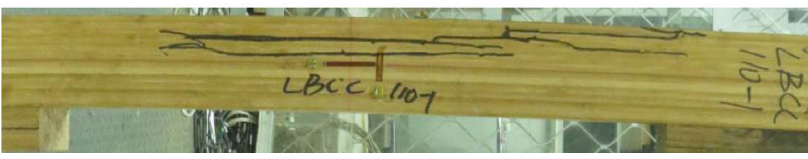

Face B

(c) Failure mode III (LBCC110-1)

Fig. 3 Typical failure modes for radial direction

As discussed above, typical failure modes along two directions are not exactly the same with each other. Even though the failure mode I for both directions is similar, the difference still can be found easily that more crack layers appeared for radial eccentric direction group specimens. The trait of more crack layers was embedded in all three modes for radial direction compared with that for the tangential direction. In addition, the main reason is that there are more bamboo strip layers for radial eccentric direction.

There are two main reasons for the failures of the specimens no matter which eccentric direction they are. In addition, the two main reasons are the bamboo joints and drill hole, particularly the first one. Bamboo joints are the weak point of the strips. Most of the specimens crack first in the bamboo joint area. However, no matter which failure reason it was, the deflection was very big when the specimen split. As can be seen from Table 1, the average

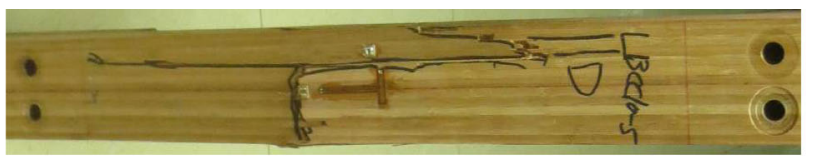

Face A

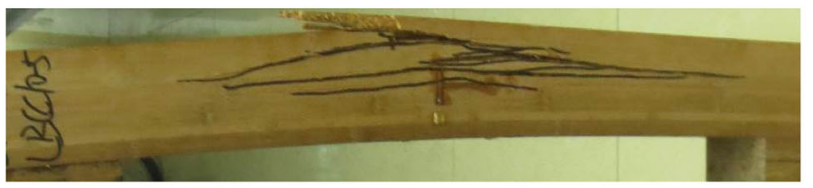

Face D

(a) Failure mode I (LBCC10-5)

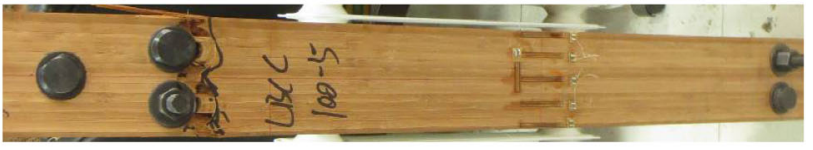

Face A

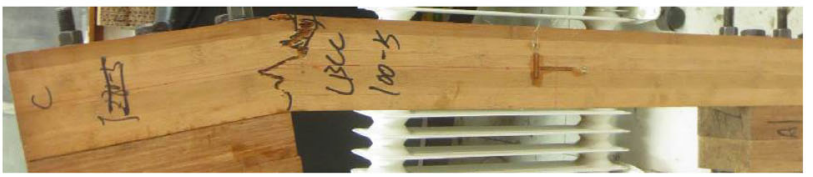

Face D

(b) Failure mode II (LBCC100-5)

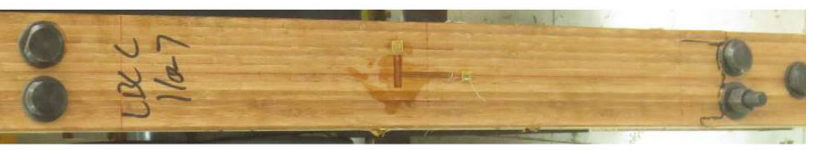

Face A

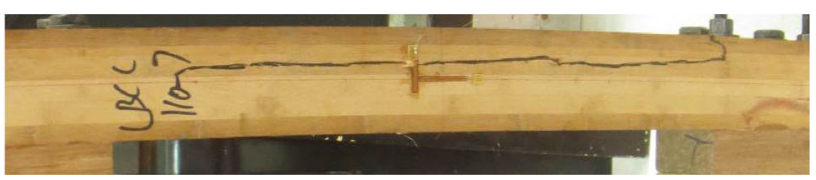

Face D

(c) Failure mode III (LBCC110-7)

Fig. 4 Typical failure modes for tangential direction

deflection at ultimate load is far bigger than the maximum allowable design value of $4.8 \mathrm{~mm}(L / 250$, where $\mathrm{L}$ is the length of the column). Two critical design criteria (deflection and strength) are always used for the structural members. Even though different failure modes happened, the column can still meet both the design criterion.

\section{Load strain curves comparison}

Typical load strain curves comparison can be seen from Figs. 5 and 6, which show how longitudinal strains and the lateral strains for the four middle side surfaces change with the increase of loading, respectively. All strains displayed an initial elastic phase. Face $\mathrm{C}$ for all specimens is always under compression condition along the longitudinal axial line no matter which eccentric direction they are. When the eccentricity value becomes bigger, the increasing speed for 
the strain value becomes bigger for both radial direction and tangential direction. The longitudinal strain values and lateral strain values for both face B and face D are always consistent during the whole loading process no matter how big the eccentricity value is and which eccentric direction they are. In addition, these two kinds of values become closer and closer to zero with the increasing of the eccentricity value which means that the mechanical properties for eccentric columns become more and more similar to that for the beam. Both the longitudinal strain values and the lateral strain values for face $\mathrm{C}$ are always the biggest among four surfaces. The ultimate longitudinal strain value on the compression surface of the specimen is biggest among four strains no matter how big the eccentricity value is and which eccentric direction they are. As defects influence the tensile strength more than compression strength, the tensile failure always happens earlier than the compression failure for the laminated bamboo column for two eccentric directions.

\section{Load lateral deflection curves comparison}

Figure 7 plots the typical lateral deflection curves comparison for two eccentric compression directions under different load grade. Similar as that for the PBSL column [3] under eccentric conditions, the variation trends are similar for the columns no matter how the test eccentricity values are and which eccentric direction they are. The sine half-wave curves have been drawn by using the dotted lines in Fig. 7. It can be seen clearly that the test curves are pretty much close to the sine line when the load value is small. When the load value becomes bigger and bigger, the curves are close to triangle more no matter how the test eccentricity values are and which eccentric direction they are. The equation of the deflection curve can be expressed as:

$w=w_{\mathrm{m}} \sin \frac{\pi H}{L}$,

where $w$ is the deflection value of the laminated bamboo lumber column, $w_{\mathrm{m}}$ is the middle deflection value of the column, $H$ is the height from the bottom to the calculation point of the column, and $L$ is the total length of the column.

\section{Strain distribution comparison}

Figures 8 and 9 plot the typical evolution of the strain profile through the loading process for the mid-span cross section of the specimens for two eccentric directions, respectively. Similar as that for the PBSL column [3] under eccentric conditions, no matter which direction it is, the strain across the cross section of the LBL column for each specimen is basically linear throughout the loading process, following standard normal section bending theory. Due to the tensile side and compression side of the LBL materials becoming plastic, the strain profile line is curved in the two ends. One phenomenon is similar for the two eccentric directions that the neutral axis position becomes higher and higher from $0 \mathrm{~mm}$ to approximately $40 \mathrm{~mm}$ with increasing of eccentricity value and then the position does not change too much for the position $40 \mathrm{~mm}$. In addition, over the course of the loading process for the specimen with small eccentricity, the neutral axis moves upwards to the middle height of the cross section, particularly during the latter part of the process. These phenomena can be seen clearly for specimen LBCC10-4 and LBCC 10-8 for each eccentric direction, respectively. However, over the course of the loading process for the specimen with large eccentricity, the neutral axis moves downwards from the centre of the column, particularly during the latter part of the process for both eccentric directions.

\section{Load middle deflection curves comparison}

Figure 10 plots the typical load against the middle deflection curves comparison for the specimens with different eccentricity under two eccentric directions. Similar as the performance for the PBSL column [3] under eccentric conditions, the load and deflection curves show that these specimens are under elastic compression in the initial stage, followed by nonlinear softening behaviour no matter which direction they

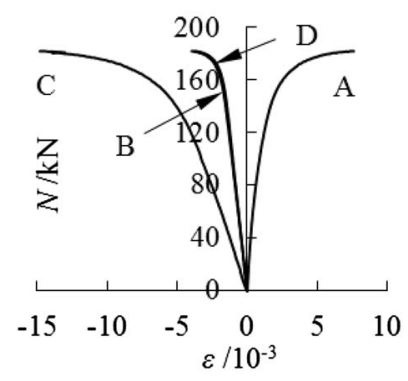

(a) $\mathrm{LBCC} 25-2$

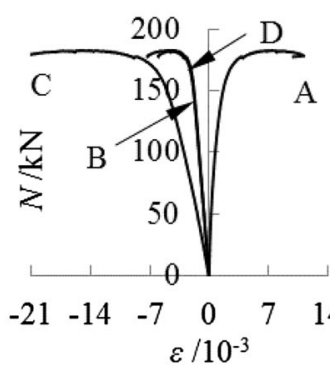

(b) LBCC 25-5

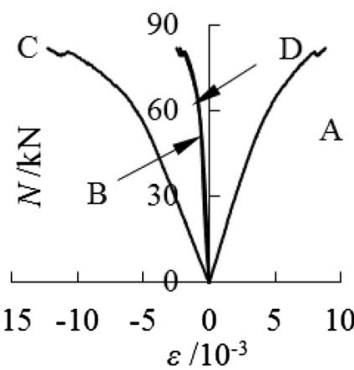

(c) LBCC 110-2

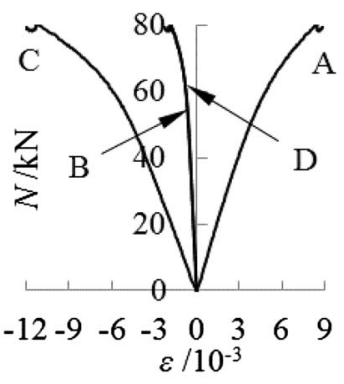

(d) LBCC 110-8

Fig. 5 Load-longitudinal strain curves comparison 


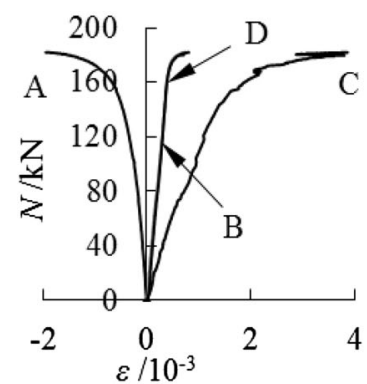

(a) $\mathrm{LBCC} 25-2$

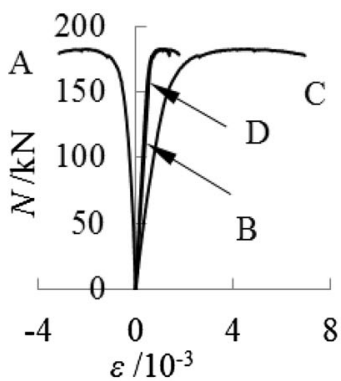

(b) LBCC 25-5

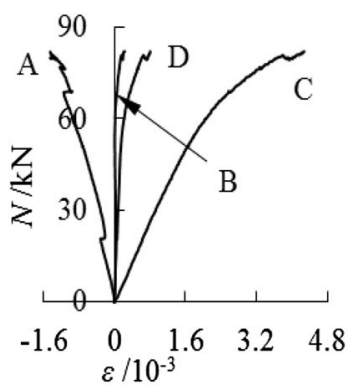

(c) LBCC 110-2

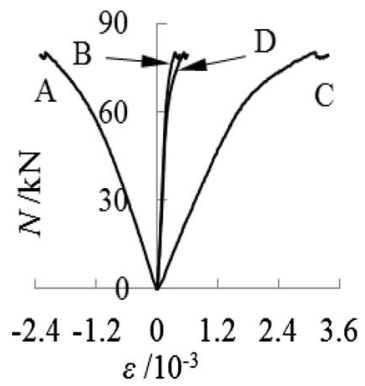

(d) LBCC 110-8

Fig. 6 Load-lateral strain curves comparison

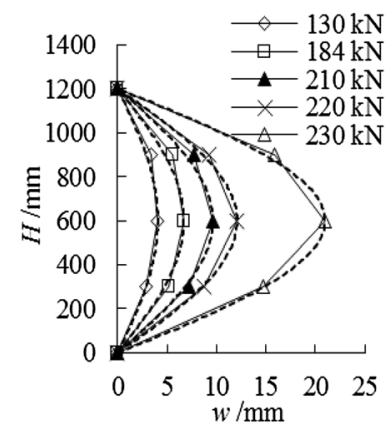

(a) $\mathrm{LBCC} 10-1$

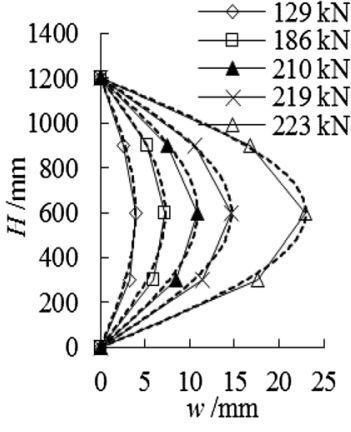

(b) $\mathrm{LBCC} 10-8$

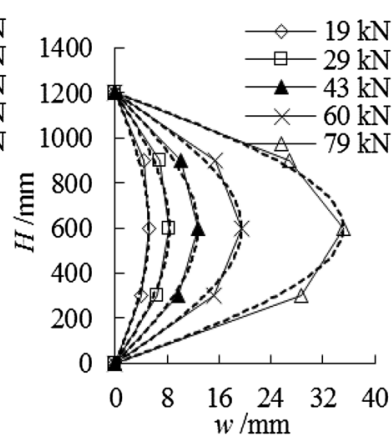

(c) $\mathrm{LBCC} 100-4$

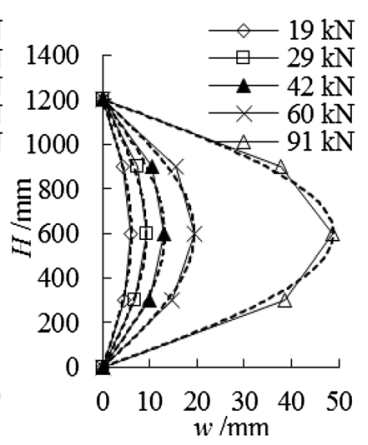

(d) LBCC 100-6

Fig. 7 Typical lateral deflection curves comparison

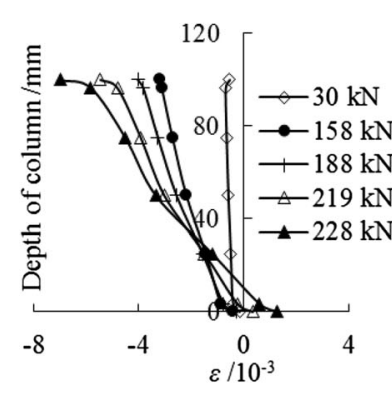

(a) LBCC10-4

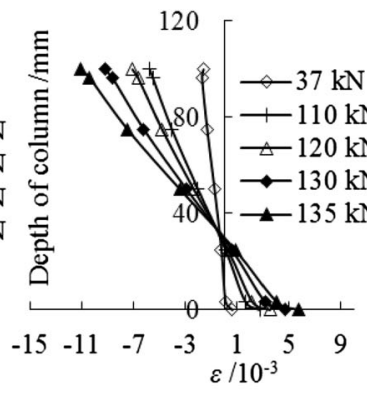

(b) LBCC40-4

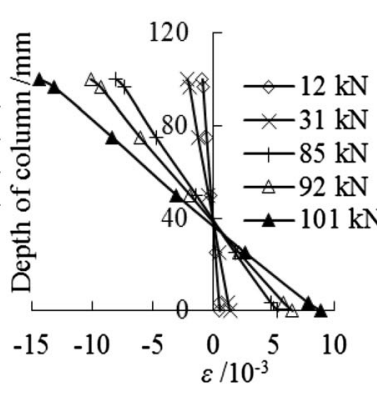

(c) $\mathrm{LBCC} 80-4$

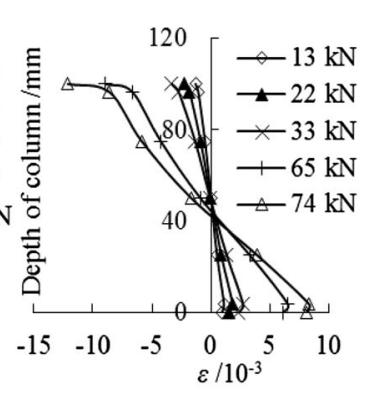

(d) LBCC110-1

Fig. 8 Typical strain profile development for radial eccentric direction

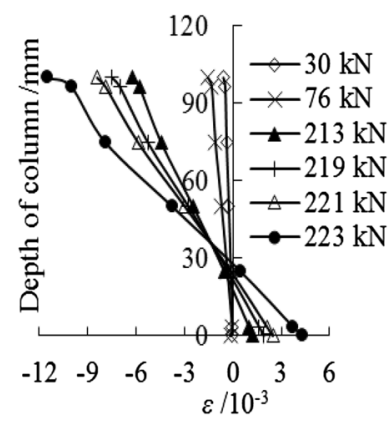

(a) $\mathrm{LBCC} 10-8$

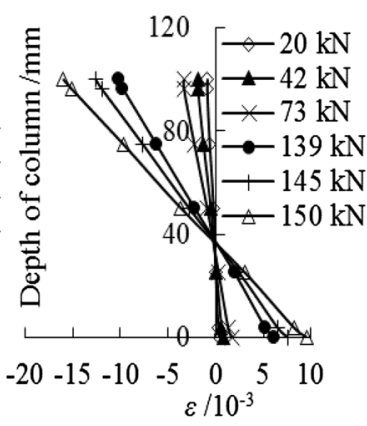

(b) LBCC40-6

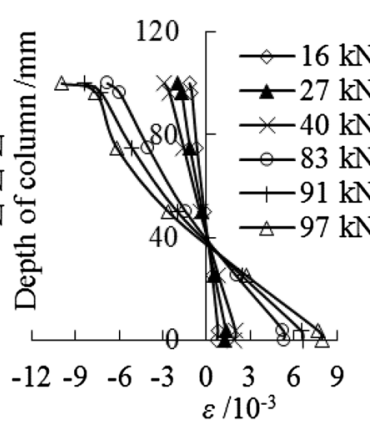

(c) LBCC $80-8$

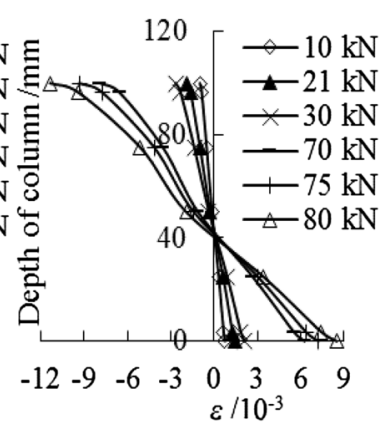

(d) $\mathrm{LBCC} 110-8$

Fig. 9 Typical strain profile development for tangential eccentric direction 
Fig. 10 Load-middle deflection curves comparison for two directions
Fig. 11 Ultimate longitudinal strain comparison for face $\mathrm{A}$

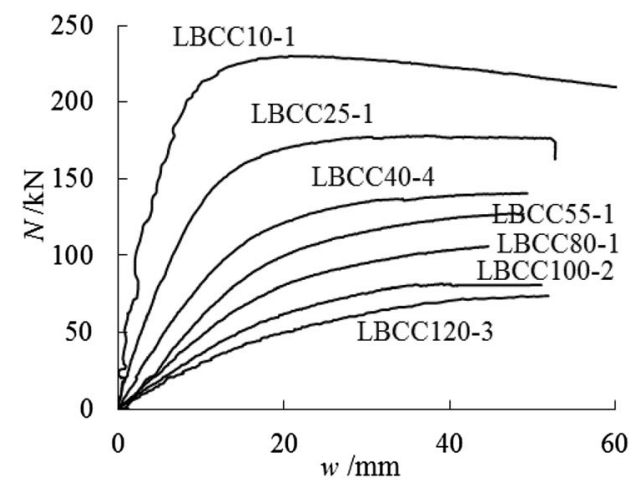

(a) Radial eccentric direction

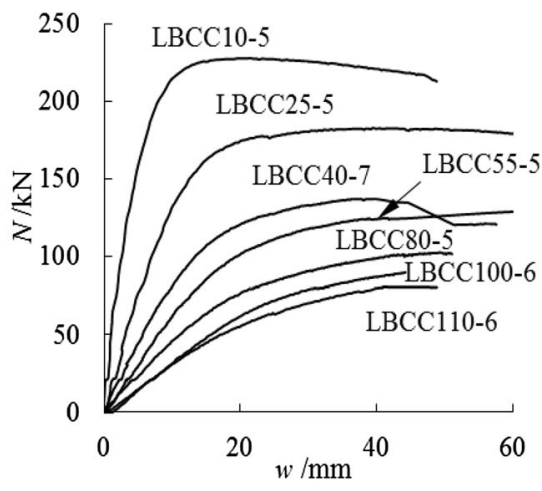

(b) Tangential eccentric direction

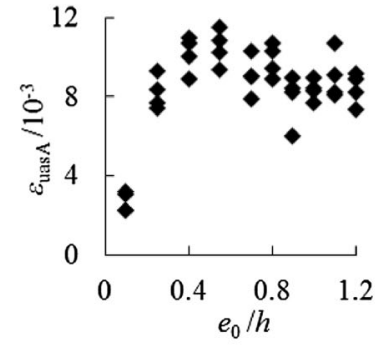

(a) Radial eccentric direction (LBL)

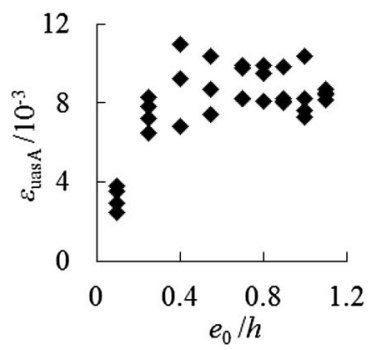

(b) Tangential eccentric direction (LBL)

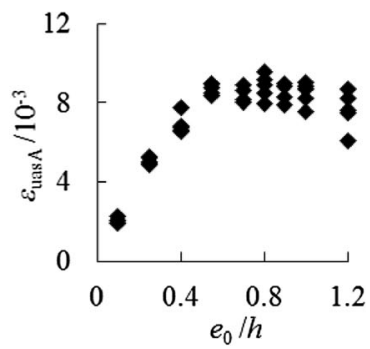

(c) PBSL
Fig. 12 Ultimate lateral strain comparison for face A

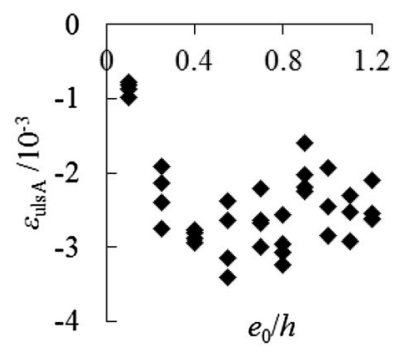

(a) Radial eccentric direction (LBL)

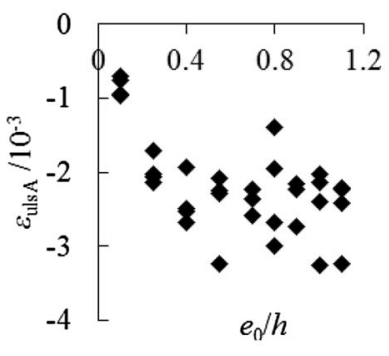

(b) Tangential eccentric direction (LBL)

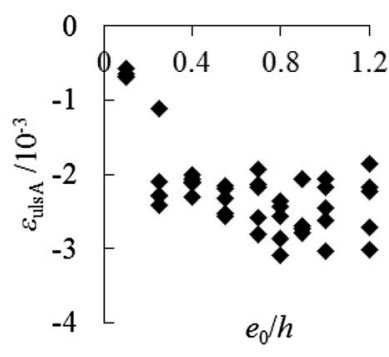

(c) PBSL are. After the peak load point, the lateral deflection increase quickly. The load is decreasing, while the displacement keeps increasing until failure happens. Purely plastic behaviour happens particular for the specimen with small eccentricity. As can be seen from Fig. 10, the lateral deflection increasing rate accelerated with the increase of the eccentricity before achieving the ultimate load. Deflection caused by the initial defects influences the bearing capacity of the specimens more and more obviously as the increase of the eccentricity ratio, and the bigger the eccentricity ratio, the bigger the deflection corresponding with the peak load.

\section{Ultimate strain comparison for three typical faces}

Figures 11 and 12 plot the ultimate longitudinal strain comparison and the ultimate lateral strain comparison for face A under two eccentric directions for LBL column and one direction for PBSL column [3], respectively. As can be seen clearly from these two figures, the variation trends are similar for both radial direction and tangential direction which are similar as that for the PBSL column. No matter which eccentric direction it is, face A undertakes tensile stress along the longitudinal direction and compressive stress along the lateral direction with increasing of the eccentricity ratio as it is tensile face. Both the ultimate longitudinal strain values and lateral strain values are small for the column specimens with small eccentricity ratio which mean that the material strength has not been brought into full play when the specimen failed under two eccentric directions. These two kinds of values increase clearly with the increasing of eccentricity ratio initially, and then do not change too much or decrease slowly. The longitudinal strain values for face A for PBSL columns are smaller than those values for the LBL columns. 
Figures 13 and 14 plot the ultimate longitudinal strain comparison and the ultimate lateral strain comparison against the eccentricity ratio for face $B$, respectively. Similar as face A, face B undertakes compressive stress along the longitudinal direction and tensile stress along the lateral direction no matter which eccentric direction and which material the specimen is. Both the absolute longitudinal strain values and the absolute lateral strain values increase with the increasing of eccentricity ratio initially, and then decrease after approximately 0.4 for the eccentricity ratio value for both two eccentric directions for LBL columns. Even though the overall trends are similar for two kinds of the materials, the absolute longitudinal strain values for PBSL columns [3] decreased after the eccentricity ratio value exceeded 0.8 which is bigger than that for LBL columns. In addition, the ultimate lateral strains values were very scattered for PBSL columns.

Figures 15 and 16 plot the ultimate longitudinal strain comparison and the ultimate lateral strain comparison against the eccentricity ratio for face $\mathrm{C}$, respectively. As compression face, face $\mathrm{C}$ undertakes compressive stress along the longitudinal direction and tensile stress along the lateral direction no matter which eccentric direction and which material the specimen is. Following the similar trends, both the longitudinal strain values and the absolute lateral strain values are small for the column specimens with small eccentricity ratio under two eccentric directions. Similar as face A, these two kinds of values increase clearly with the increasing of eccentricity ratio initially, and then do not change too much or decrease slowly for both eccentric directions. The strain values for LBL columns decreased earlier than those for PBSL columns [3].

\section{Combined analysis}

\section{Ultimate displacement comparison}

Figures 17 and 18 plot the ultimate middle deflection comparison and ultimate axial deformation comparison for
Fig. 13 Ultimate longitudinal strain comparison for face B

Fig. 14 Ultimate lateral strain comparison for face B

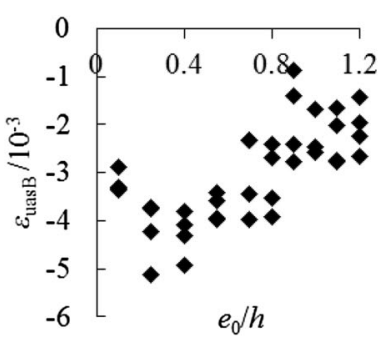

(a) Radial eccentric direction (LBL)

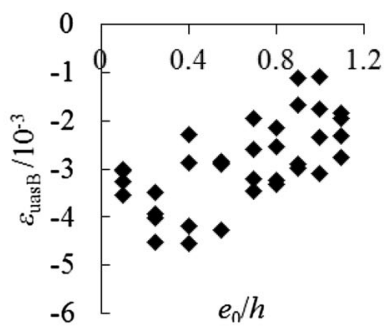

(b) Tangential eccentric direction (LBL)

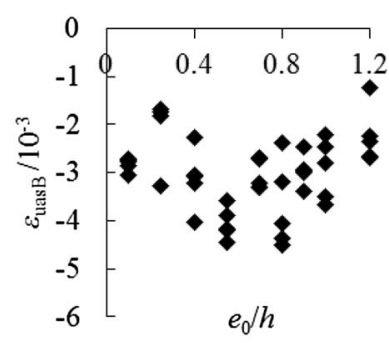

(c) PBSL

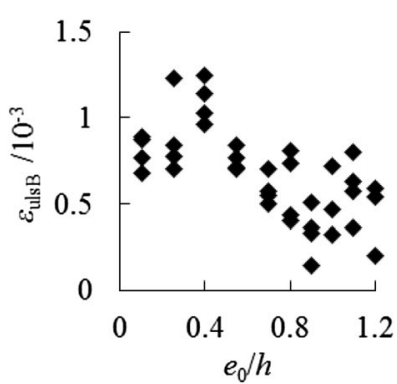

(a) Radial eccentric direction (LBL)

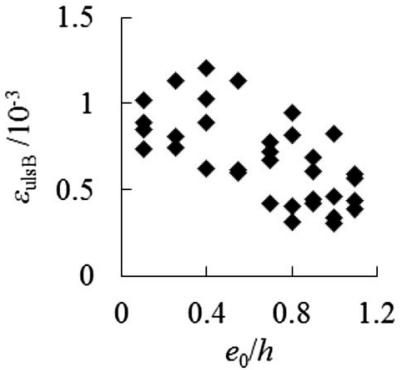

(b) Tangential eccentric direction (LBL)

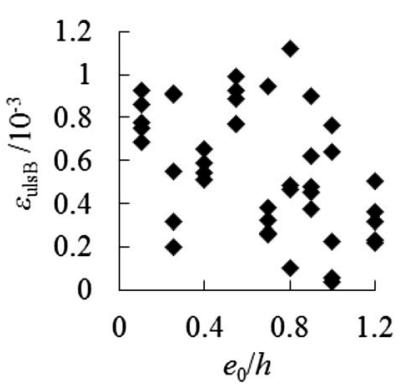

(c) PBSL
Fig. 15 Ultimate longitudinal strain comparison for face C. Load relationship comparison

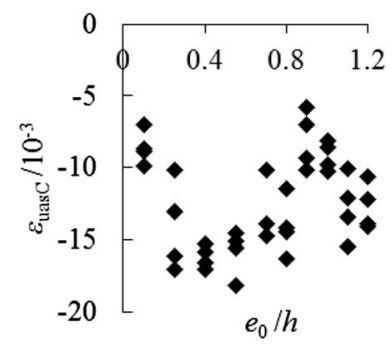

(a) Radial eccentric direction (LBL)

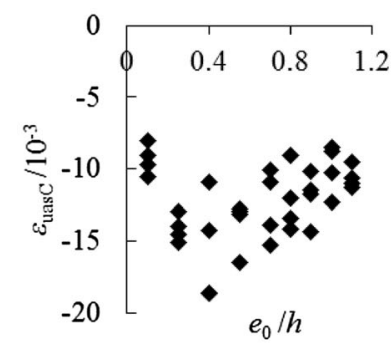

(b) Tangential eccentric direction (LBL)

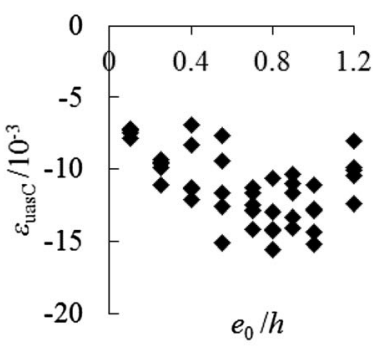

(c) PBSL 
Fig. 16 Ultimate lateral strain comparison for face $\mathrm{C}$

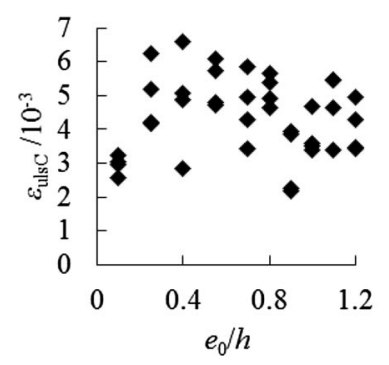

(a) Radial eccentric direction (LBL)

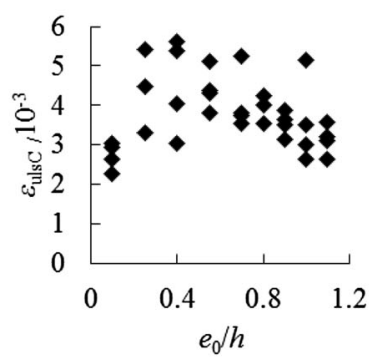

(b) Tangential eccentric direction (LBL)

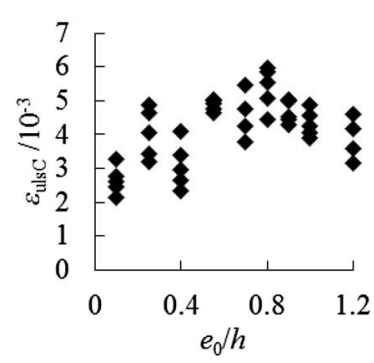

(c) PBSL
Fig. 17 Ultimate middle deflection comparison

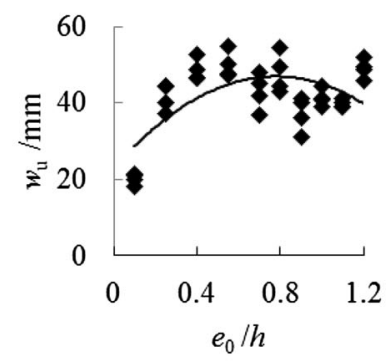

(a) Radial eccentric direction (LBL)

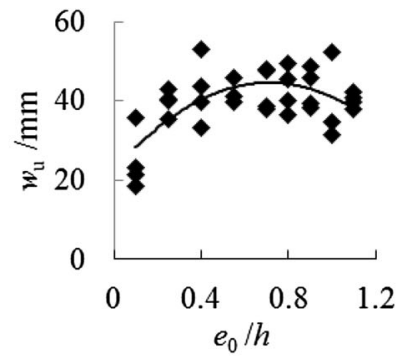

(b) Tangential eccentric direction (LBL)

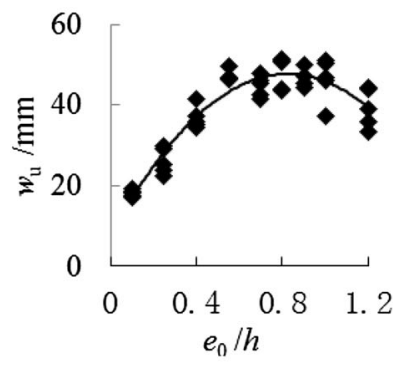

(c) PBSL
Fig. 18 Ultimate axial deformation comparison

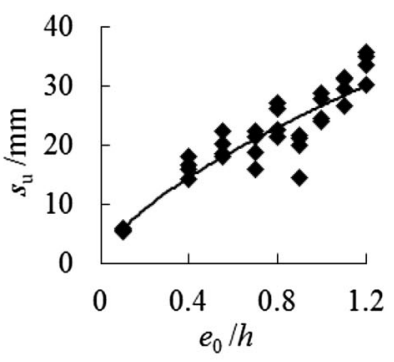

(a) Radial eccentric direction (LBL)
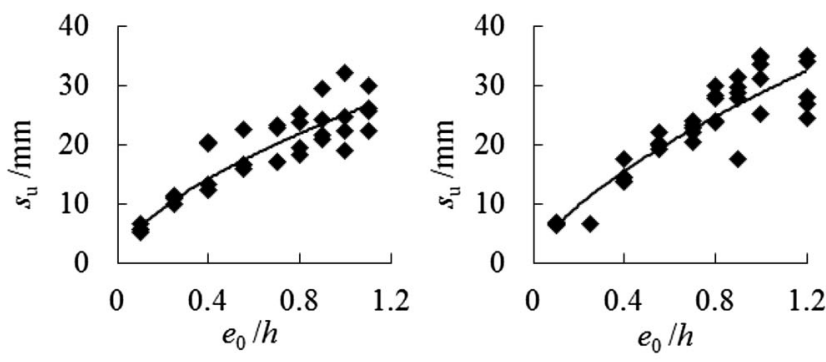

(c) PBSL

(b) Tangential eccentric direction (LBL)
LBL columns under two eccentric directions and for PBSL columns [3] with different eccentricity ratio. As can be seen from Fig. 17, the ultimate middle deflection values become bigger and bigger with the increase of the eccentricity ratio as a whole and then they do not change too much or decrease slightly. However, the ultimate axial deformation keeps increasing with the increasing of eccentricity ratio, as shown in Fig. 18, which is different from the ultimate middle deflection for both two eccentric directions. Similar as the absolute values for the strains, the ultimate middle deflection values for PBSL columns keep increasing for a longer time than that for LBL columns.

Based on the test results, the relationship between ultimate middle deflection and eccentricity ratio, under the conditions mentioned previously, can be expressed as follows, respectively:

$$
\begin{aligned}
& w_{\mathrm{ur}}=-39.9\left(e_{0} / h\right)^{2}+62\left(e_{0} / h\right)+22.8, \\
& w_{\mathrm{ut}}=-43.5\left(e_{0} / h\right)^{2}+62\left(e_{0} / h\right)+22.5,
\end{aligned}
$$

$w_{\mathrm{us}}=-58.7\left(e_{0} / h\right)^{2}+96.6\left(e_{0} / h\right)+7.9$,

where $w_{\mathrm{ur}}$ and $w_{\mathrm{ut}}$ are the ultimate middle deflection of the LBL column for radial eccentric direction and tangential eccentric direction, respectively $(\mathrm{mm}), w_{\mathrm{us}}$ is the ultimate middle deflection of the PBSL column (mm), $e_{0}$ is the eccentricity value of the PBSL column ( $\mathrm{mm})$, and $h$ is the height along the eccentric direction of the cross section (mm).

According to the test results for the specimens under the conditions mentioned previously, the relationship between ultimate axial deformation and eccentricity ratio can be expressed as:

$s_{\mathrm{ur}}=26.6\left(e_{0} / h\right)^{0.663}$

$s_{\mathrm{ut}}=25.1\left(e_{0} / h\right)^{0.618}$

$s_{\mathrm{us}}=28.7\left(e_{0} / h\right)^{0.67}$

where $S_{\text {ur }}$ and $S_{\text {ut }}$ are the ultimate axial deformation of the LBL column for radial eccentric direction and tangential 
Fig. 19 Ultimate load comparison

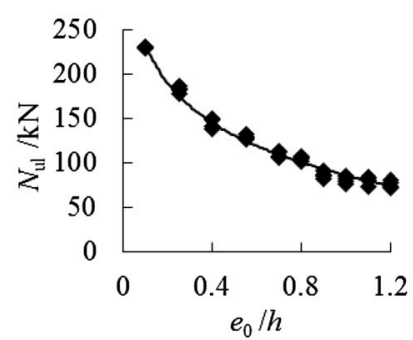

(a) Radial eccentric direction (LBL)

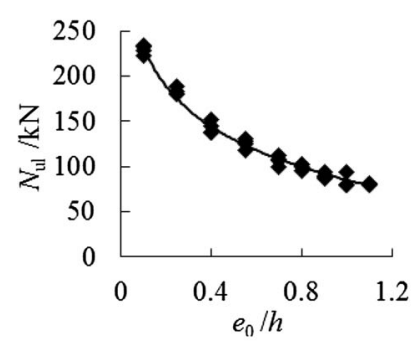

(b) Tangential eccentric direction (LBL)

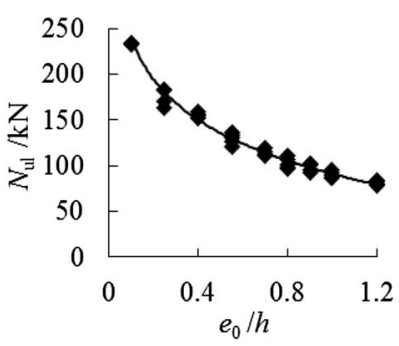

(c) PBSL
Fig. 20 Ultimate moment comparison

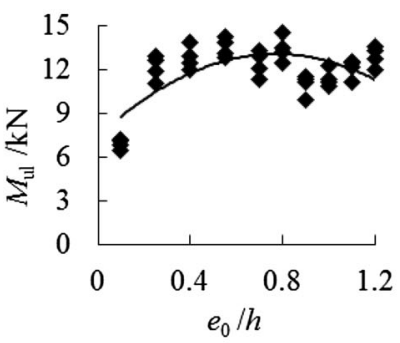

(a) Radial eccentric direction (LBL)

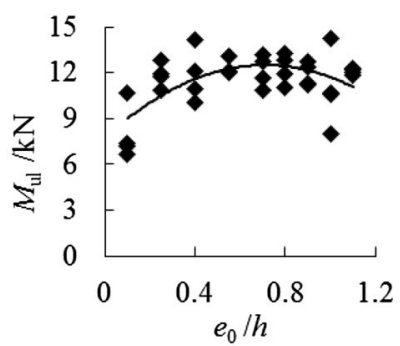

(b) Tangential eccentric direction (LBL)

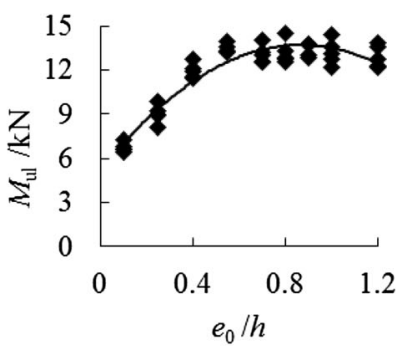

(c) PBSL eccentric direction, respectively $(\mathrm{mm})$, and $S_{\mathrm{us}}$ is the ultimate axial deformation of the PBSL column ( $\mathrm{mm})$.

\section{Ultimate bearing capacity comparison}

Figures 19 and 20 plot the ultimate load comparison and the ultimate moment comparison for columns with different eccentricity ratios for ultimate load under two eccentric directions. The ultimate load values decrease quickly with the increase of the eccentricity ratio in the beginning, and then decrease slowly. However, the ultimate moment increases first and then do not change too much or decrease slightly. The eccentricity ratio is the main influencing factors on the bearing capacity of the columns. The relationship between ultimate load and eccentricity ratio, under the conditions mentioned previously, can be expressed as:

$N_{\text {ulr }}=86.14-64.3 \ln \left(e_{0} / h\right)$,

$N_{\text {ult }}=85.12-64.6 \ln \left(e_{0} / h\right)$,

$N_{\mathrm{uls}}=91.13-64.3 \ln \left(e_{0} / h\right)$,

where $N_{\text {ulr }}$ and $N_{\text {ult }}$ are the ultimate bearing capacity of the LBL column for radial eccentric direction and tangential eccentric direction, respectively $(\mathrm{kN})$, and $N_{\mathrm{ul}}$ is the ultimate bearing capacity of the PBSL column $(\mathrm{kN})$.

On the basis of the test results, the relationship between ultimate load and eccentricity ratio, under the conditions mentioned previously, can be expressed as:

$M_{\mathrm{ulr}}=-9.46\left(e_{0} / h\right)^{2}+14.6\left(e_{0} / h\right)+7.41$,
$M_{\mathrm{ult}}=-9.45\left(e_{0} / h\right)^{2}+13.4\left(e_{0} / h\right)+7.74$,
$M_{\mathrm{uls}}=-11.4\left(e_{0} / h\right)^{2}+19.8\left(e_{0} / h\right)+5.12$,

where $M_{\mathrm{ulr}}$ and $M_{\mathrm{ult}}$ are the ultimate moment of the LBL column for radial eccentric direction and tangential eccentric direction, respectively $(\mathrm{kN})$, and $M_{\mathrm{ul}}$ is the ultimate moment of the PBSL column $(\mathrm{kN})$.

Figure 21 plots the ultimate moment and load relationship comparison for the columns. It can be seen clearly that there is one limit state for all three figures. The trends for $M_{\mathrm{ul}}-N_{\mathrm{ul}}$ curves are similar as that for reinforced concrete columns under eccentric condition. For the specimen with smaller eccentricity values, the $N_{\mathrm{ul}}$ values for LBL columns decrease more quickly with the increasing of $M_{\mathrm{ul}}$ than the $N_{\mathrm{ul}}$ values for PBSL columns.

As discussed above, the mechanical properties for two eccentric directions are similar with each other even though the failure modes are somewhat not very same for LBL columns. That is to say, they could follow the same design rules when used in the construction area. While even though the mechanical properties are similar for both LBL columns and PBSL columns [3] as a whole, there are still some clear performance differences between the two kinds of materials. It might be not proper to ignore the difference when designing. Anyway, they have different inner structures and production processes and technologies with each other.

\section{Calculation for the ultimate bearing capacity}

The eccentricity influencing coefficient $\varphi_{\mathrm{e}}$ for calculating the ultimate bearing capacity can be expressed as follows: 
Fig. 21 Ultimate moment and load relationship comparison

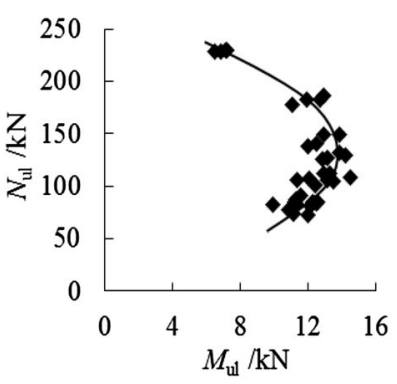

(a) Radial eccentric direction (LBL)

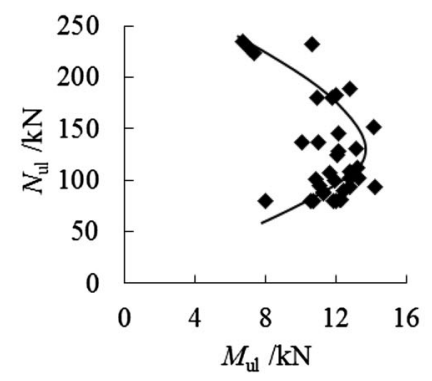

(b) Tangential eccentric direction (LBL)

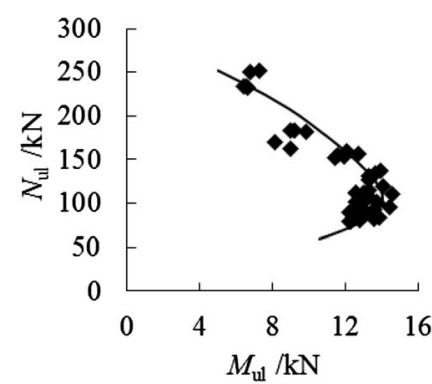

(c) PBSL

Table 2 Comparison between the test results and calculation results

\begin{tabular}{lcllllll}
\hline Group & $e_{0} / h$ & $\varphi_{\mathrm{e}}$ & $N_{\mathrm{ul}}^{\mathrm{t}}(\mathrm{kN})$ & $N_{\mathrm{ul}}^{\mathrm{c}}(\mathrm{kN})$ & $\mu$ & $\mathrm{SD}$ & $\mathrm{COV}$ \\
\hline LBCC10 & 0.10 & 0.466 & 229.3 & 231.7 & 0.99 & 0.0141 & 0.0142 \\
LBCC25 & 0.25 & 0.362 & 182.7 & 179.7 & 1.017 & 0.0186 & 0.0183 \\
LBCC40 & 0.4 & 0.295 & 143.6 & 146.7 & 0.979 & 0.041 & 0.0419 \\
LBCC55 & 0.55 & 0.250 & 126.8 & 124.0 & 1.023 & 0.0351 & 0.0343 \\
LBCC70 & 0.7 & 0.216 & 108.2 & 107.4 & 1.008 & 0.0384 & 0.0381 \\
LBCC80 & 0.8 & 0.198 & 102.2 & 98.54 & 1.037 & 0.0398 & 0.0384 \\
LBCC90 & 0.9 & 0.183 & 87.93 & 91.06 & 0.966 & 0.0405 & 0.0419 \\
LBCC100 & 10 & 0.170 & 81.90 & 84.64 & 0.968 & 0.0608 & 0.0628 \\
LBCC110 & 1.1 & 0.159 & 80.47 & 79.06 & 1.018 & 0.0408 & 0.0401 \\
LBCC120 & 1.2 & 0.149 & 76.18 & 74.18 & 1.027 & 0.0516 & 0.0503 \\
\hline
\end{tabular}

$S D$ standard deviation, $C O V$ coefficients of variation

$\varphi_{\mathrm{e}}=N_{\mathrm{ul}} / N_{0}$,

where $N_{\mathrm{ul}}$ is the ultimate bearing capacity of LBL columns under eccentric compression, and $\mathrm{N}_{0}$ the ultimate bearing capacity of LBL columns under axial compression.

In reality, there is interaction between the eccentricity influencing coefficient and geometric parameters of the laminated bamboo lumber columns. Combining with the numerical analysis and then reevaluating the constant coefficients by statistical regression on the whole test data set, an equation for calculating the eccentricity influencing coefficient $\varphi_{\mathrm{e}}$ of laminated bamboo lumber columns can be expressed as:

$\varphi_{\mathrm{e}}=\frac{1}{1.73+4.14 e_{0} / h}$,

where $e_{0}$ is the eccentricity value of the LBL column, and $h$ is the height along the eccentric direction of the cross section.

According to formula (14), the ultimate bearing capacity can be calculated by the following equation:

$N_{\mathrm{ul}}=\varphi_{\mathrm{e}} N_{0}$,

where the stability coefficient $\varphi_{\mathrm{e}}$ can be calculated by Eq. (15).

The test results and calculation results by Eq. (16) for each group of specimens, in the form of mean, standard deviation (SD), and coefficients of variation (COV) values, are summarised in Table 2. $N_{\mathrm{ul}}^{\mathrm{t}}$ is the average test ultimate load and $N_{\mathrm{ul}}^{\mathrm{c}}$ is the calculation ultimate load by Eq. (16). $\mu$ stands for the mean value of $N_{\mathrm{ul}}^{\mathrm{t}} / N_{\mathrm{ul}}^{\mathrm{c}}$. It can be seen clearly that the standard deviation coefficients for most of groups are no more than 0.052 except one group with the eccentricity value of $100 \mathrm{~mm}$. The standard deviation coefficient for group LBCC100 is 0.0608 which is also not very big. In addition, most of the coefficients of variation are less than 0.051 except group LBCC100 with the value of 0.0628. As a whole, all these two kinds of values are small. That is to say, the calculation results obtained from the equation give good agreement with the test results.

All the equations presented in this paper show how the factors investigated were found to influence the mechanical performance of columns under eccentric compression in this particular series of the tests, and give reference for further work to establish a generally applicable formula for code adoption.

\section{Conclusions}

To investigate the mechanical properties of laminated bamboo lumber column under radial and tangential eccentric compression, 80 column tests have been 
performed. Based on the analysis of the test data, the following conclusions can be drawn.

1. Even though three typical failure modes can be divided for both two eccentric directions, they are not exactly the same with each other. More crack layers appeared for radial eccentric direction group specimens due to the layer structure.

2. There are two main reasons which are the bamboo joints and drill hole for the failures of the specimens under two eccentric directions, particularly the first one. The tensile failure always happens earlier than the compression failure for the laminated bamboo as defects influence the tensile strength more than compression strength.

3. No matter which eccentric directions they are, the lateral deflection curves are close to the sine line, and the strain across the cross section of the laminated bamboo lumber column for each specimen is basically linear throughout the loading process, following standard normal section bending theory.

4. The mechanical properties for two eccentric directions are similar with each other. The ultimate strain values variation trend are similar for both two eccentric directions, so are the ultimate displacement values and the ultimate bearing capacity values. Thus, they could follow the same design rules when used in the construction area.

5. Even though the mechanical properties are similar for both LBL columns and PBSL columns as a whole, there are still some clear performance differences between the two kinds of materials.

6. Combined all the test data under two eccentric directions, an equation for calculating the radial eccentricity influencing coefficient $\varphi_{\mathrm{e}}$ of LBL columns is proposed. The calculation results obtained from the equations give good agreement with the test results.

Acknowledgments The material presented in this paper is based upon work supported by the National Natural Science Foundation of China (51308301), the Natural Science Foundation of Jiangsu Province (No. BK20130978), the China Postdoctoral Science Foundation (2015M580382), Jiangsu Postdoctoral Science Foundation Project (1501037A), Open Fund Project from Key Laboratory of Concrete and Pre-stressed Concrete Structure of Ministry of Education (Southeast university) and a Project Funded by the Priority Academic Program Development of Jiangsu Higher Education Institutions. Any opinions, findings, and conclusions or recommendations expressed in this material are those of the writer(s) and do not necessarily reflect the views of the foundations. The writers gratefully acknowledge Nian-qiang ZHOU, Qi-jun WANG, Zhuang-yan SHEN, Hao YANG, Ming-lei YAO, Shuai-hong ZHANG, Jin-yuan WANG, Zhi-hao YIN, Jin-xiu SUN, Wei-xu ZHU, and others from the Nanjing Forestry University for helping with the tests.

\section{References}

1. Xiao Y, Shan B (2013) Morden bamboo structures. China Architecture and Building Press, Beijing

2. Wu WQ (2014) Experimental analysis of bending resistance of bamboo composite I-shaped beam. J Bridge Eng 19(4):04013014$1-13$

3. Li HT, Su JW, Deeks AJ, Zhang QS, Wei DD, Yuan CG (2015) Eccentric compression performance of parallel bamboo strand lumber column. BioResources 10:7065-7080

4. Chen G, Li HT, Zhou T, Li CL (2015) Experimental evaluation on mechanical performance of OSB webbed parallel strand bamboo I-Joist with holes in the web. Constr Build Mater 101:91-98

5. Nugroho N, Ando N (2001) Development of structural composite products made from bamboo II fundamental properties of laminated bamboo lumber. J Wood Sci 47:237-242

6. Sulaiman O, Hashim R, Wahab R (2006) Evaluation of shear strength of oil treated laminated bamboo. Bioresour Technol 97:2466-2469

7. Katleen DF, Ronald R (2009) One laminated bamboo-frame house per hectare per year. Constr Build Mater 23:210-218

8. Paes JB, Oliveira AKF, Oliveira E (2009) Physical-mechanical characterization of the glue laminated bamboo (Dendrocalamus giganteus). Ciencia Florestal 19:41-51

9. Sulastiningsih IM (2009) Physical and mechanical properties of laminated bamboo board. J Tropic Forest Sci 21:246-251

10. Correal JF, Ramire LF (2010) Adhesive bond performance in glue line shear and bending for glued laminated guadua bamboo. J Tropic Forest Sci 22:433-439

11. Mahdavi M, Clouston PL, Arwade SR (2012) A low-technology approach toward fabrication of laminated bamboo lumber. Constr Build Mater 29:257-262

12. Lee CH, Chung MJ, Lin CH, Yang TH (2012) Effects of layered structure on the physical and mechanical properties of laminated moso bamboo (Phyllosachys edulis) flooring. Constr Build Mater 28:31-35

13. Li HT, Deeks AJ, Zhang QS, Gang W (2016) Flexural performance of laminated bamboo lumber Beam. Bio Resour 11:929-943

14. Verma CS, Chariar VM (2012) Development of layered laminate bamboo composite and their mechanical properties. Compos Part B Eng 43:1063-1069

15. Verma CS, Chariar VM (2013) Stiffness and strength analysis of four layered laminate bamboo composite at macroscopic scale. Compos part B Eng 45:369-376

16. Verma CS, Kr Sharma Naresh, Chariar VM, Maheshwari S, Hada MK (2014) Comparative study of mechanical properties of bamboo laminae and their laminates with woods and wood based composites. Compos Part B Eng 60:523-530

17. Correal JF, Juan SE, Fernando R, Luis EY (2014) Experimental evaluation of physical and mechanical properties of glued laminated Guadua angustifolia Kunth. Constr Build Mater 73:105-112

18. Sharma B, Gatóo A, Michael H (2015) Effect of processing methods on the mechanical properties of engineered bamboo. Constr Build Mater 83:95-101

19. Yeh MC, Lin Y (2012) Finger joint performance of structural laminated bamboo member. J Wood Sci 58:120-127

20. Lee AWC, Bai XS, Bangi AP (1998) Selected properties of laboratory-made laminated-bamboo lumber. Holzforschung 52:207-210

21. Wei Y, Jiang ShX, Lv QF, Zhang QS, Wang LB, Lv ZT (2011) Flexural performance of glued laminated bamboo beams. Adv Mater Res 168-170:1700-1703 
22. Lima DM, Amorim MM, Lima JHC, Barbosa NP, Wilrich FL (2014) Behavior analysis of glued laminated bamboo beam under bending. Ambiente Construído 14:15-27

23. Sinha A, Way D, Mlasko S (2014) Structural performance of glued laminated bamboo beams. J Struct Eng 140:040130211-04013021-8

24. Li Z, He MJ, Tao D, Li M (2016) Experimental buckling performance of scrimber composite columns under axial compression. Compos Part B Eng 86:203-213

25. Taheri F, Nagaraj M, Khosravi P (2000) Buckling response of glue-laminated columns reinforced with fiber-reinforced plastic sheets. Compos Struct 50:207-216

26. Li HT, Zhang QS, Huang DD, Deeks AJ (2013) Compressive performance of laminated bamboo. Compos Part B Eng $54: 319-328$
27. Su JW, Li HT, Yang P, Zhang QS, Chen G (2015) Mechanical Performance Study on laminated bamboo lumber column pier under axial compression. China Forestry Science and Technology 29:89-93

28. Luna P, Takeuchi C, Alvarado C, Moreno I (2013) Glued laminated Guadua angustifolia bamboo columns. Acta Horticulturae, Int Soc Horti Sci Belgium, pp 125-129

29. Li HT, Su JW, Zhang QS, Deeks AJ, Hui D (2015) Mechanical performance of laminated bamboo column under axial compression. Compos Part B Eng 79:374-382

30. Li HT, Chen G, Zhang QS, Ashraf M, Xu B, Li YJ (2016) Mechanical properties of laminated bamboo lumber column under radial eccentric compression. Constr Build Mater 121:644-652 